\title{
OTIOTOMICS
}

Revista de economía, empresa y sociedad

TRABAJO FINAL DE GRADO (TFG). PLAN DE EMPRESA

\section{Classyfied, la compra más fácil}

\section{Daniel Macías Perea}

Alumni grado ADE (UOC)

RESUMEN ¿Cada vez que visitas el supermercado compras los mismos productos? ¿Estás harto de desaprovechar las pocas horas de tiempo libre que tienes en una tarea tan repetitiva y monótona? ¿Acabas invirtiendo el mismo tiempo en hacer la compra en línea que visitando un supermercado tradicional?

En este artículo se presenta una síntesis del plan de empresa de Classyfied, elaborado como trabajo final del grado ${ }^{1}$ de Administración y Dirección de Empresas. Su modelo de negocio pretende resolver todas las insatisfacciones que produce el proceso de compra de productos de alimentación, proponiendo una modernización de la cadena de distribución mediante la inclusión de nuevas tecnologías en el envasado y en el hogar de los consumidores finales.

Classyfied, como se verá al largo de este texto, demuestra ser una idea de negocio viable y rentable económicamente, y ofrece propuestas claras mediante un producto innovador y realista que supondrá una revolución en la industria de la distribución de alimentos al por menor.

PALABRAS CLAVE distribución al por menor de alimentos; reposición automática; RFID; patrones de consumo; reciclaje; venta en línea

\section{Classyfied, the easiest purchase}

ABSTRACT Do you end up buying the same products every time you go to the supermarket? Are you tired of wasting your leisure time in a repetitive and monotonous task such as buying groceries? Do you use the same amount of time online shopping as going to the supermarket?

This article presents a synthesis of Classyfied's Business Plan, written as the Final Thesis of the Degree in Business Administration. Its business model will solve all dissatisfaction caused by the shopping process by upgrading the distribution chain and introducing new technologies in the packaging and at the consumers' home.

1. Depositado en el Repositorio Institucional de la UOC (O2) http://hdl.handle.net/10609/42481, el portal que recoge, difunde y preserva las publicaciones digitales en acceso abierto de los miembros de la UOC, elaboradas en el desarrollo de sus actividades de investigación, de docencia y de gestión. 
Classyfied, as it will be introduced in this text, proves to be a feasible and profitable business idea, offering clear proposals by means of an innovative and realistic product that will entail a revolution in the food retail industry.

KEYWORDS food retail; automatic reposition; RFID; consumption Pattern; recycling; online selling

\section{Introducción y contexto}

Según el último estudio del Observatorio del Consumo y la Distribución Alimentaria (1), dependiente del Ministerio de Agricultura, Alimentación y Medio Ambiente, el tiempo medio destinado a la compra de productos de alimentación por hogar supera las tres horas semanales ( 3 horas y 1 minuto), cifra que se ha incrementado desde el inicio de la crisis en más de un 10\%.

En la misma línea, la encuesta más reciente de Uso del Tiempo del Instituto Nacional de Estadística (INE) (2) afirma que, de media, en España se dedican $\mathbf{2 6}$ minutos diarios a actividades de compras y servicios. Además, el estudio muestra una clara reducción de las horas dedicadas al tiempo libre (vida social, deportes, aficiones, etc.). La diferencia se acentúa en el segmento de personas ocupadas, habitantes de capitales de provincia, edad comprendida entre los 24 y los 44 años y con estudios superiores finalizados. Este grupo representa, como veremos, el perfil del usuario potencial de Classyfied.

Adicionalmente, las familias están experimentando una reducción del poder adquisitivo sin comparación en las últimas décadas. Según las estadísticas anuales del INE (3), el gasto medio por hogar fue en 2013 de $27.908 €$, un 3,7\% inferior al del 2012, con reducciones del 1\% en el grupo de alimentación y bebidas no alcohólicas. Este hecho está promoviendo la aparición de una nueva tendencia en la sociedad: la compra inteligente, que combina una mayor exigencia del consumidor en la búsqueda de la excelencia de los productos adquiridos, optimizando el tiempo y los recursos destinados a su adquisición.

En línea con los dos puntos anteriores, el comercio electrónico ha demostrado ser capaz de adaptarse a las nuevas necesidades de los compradores, ofreciéndoles mejores precios y flexibilizando el tiempo de compra. De esta forma, el peso de internet en el proceso de compra ha crecido año tras año en la última década. Analizando solo el grupo de bienes de consumo de movimiento rápido se detecta un crecimiento en el $\mathbf{2 0 1 3}$ de la venta en línea del $\mathbf{3 1}$ \% según un reciente estudio de la consultora Kantar (4).

Por otro lado, es de destacar la importancia que está obteniendo el internet de las cosas, es decir, el concepto de interconexión digital de los objetos cotidianos con internet. Esta revolución tecnológica, que está por desarrollarse plenamente aún, tiene un potencial de crecimiento muy elevado. Los expertos en la materia consideran que su impacto alterará el modo como nos relacionamos con internet y estiman que los ingresos generados por este mercado estarán entre los cuatro y los once trillones de dólares en 2025 (5).

Finalmente, un último punto del entorno que destacar es la cada vez mayor conciencia en el cuidado del medio ambiente, más concretamente referida al reciclaje de productos domésticos. Aunque las ciudades españolas ya disponen de contenedores preparados para la separación de residuos, queda mucho por mejorar, fundamentalmente en el buen uso de estos. La encuesta de recogida y tratamiento de residuos urbanos del INE destaca que en 2012 (6), pese a recoger un 3,8\% menos de residuos a nivel global, la cantidad de residuos separados se redujo en menor proporción respecto a los residuos no separados.

En este contexto, la optimización de una de las tareas domésticas más consumidoras de tiempo, como es la compra de productos de alimentación, mediante la aplicación de tecnología puntera sobre un proceso de compra en línea mientras se potencia el reciclaje cobra pleno sentido y se decide desarrollar esta idea de negocio como TFG.

El objetivo global de este trabajo es analizar la posibilidad de revolucionar la cadena de distribución de alimentos y bebidas al por menor mediante la inclusión de nuevas tecnologías en el envasado y en el hogar de los consumidores finales. Es decir, con el TFG desarrollado se pretende profundizar en la rentabilidad econó- 
mica de un nuevo producto que se situaría en el hogar de los consumidores y que, al detectar los alimentos y bebidas desechados, rompería con la tradicional relación entre distribuidores minoristas de alimentos (supermercados, hipermercados, etc.), productores y consumidores, ofreciendo ventajas para todas las partes.

\section{Diseño y desarrollo de la idea de negocio}

\section{Papelera de reciclaje con reconocimiento automático de productos}

La pieza fundamental de esta idea de negocio que supondrá una disrupción en la distribución de productos de alimentación es un sistema único que permite identificar de forma automática los productos que son consumidos en el hogar. Esta identificación se realizará mediante una papelera de reciclaje doméstica equipada con tecnologías punteras de comunicación sin hilos (Wi-Fi/3G) y lectores de etiquetas de radiofrecuencia (identificación por radiofrecuencia o RFID). Esta papelera, diseñada y producida por Classyfied, captura información sobre los productos consumidos en el preciso momento de ser reciclados al ser detectado su envase, y transmite esta información instantáneamente a los servidores de Classyfied mediante la conexión a internet disponible en el domicilio del usuario. Para la identificación, los envases de los productos contendrán una etiqueta RFID, las cuales tienden a sustituir a los actuales códigos de barras, lo que permite el intercambio de información sin contacto visual y a un coste muy reducido. Dado que este sistema es el principal valor añadido de la empresa, se ha cursado una solicitud de patente con el fin de protegerse frente a futuros competidores.

Figura 1. Diseño de la PRA de Classyfied

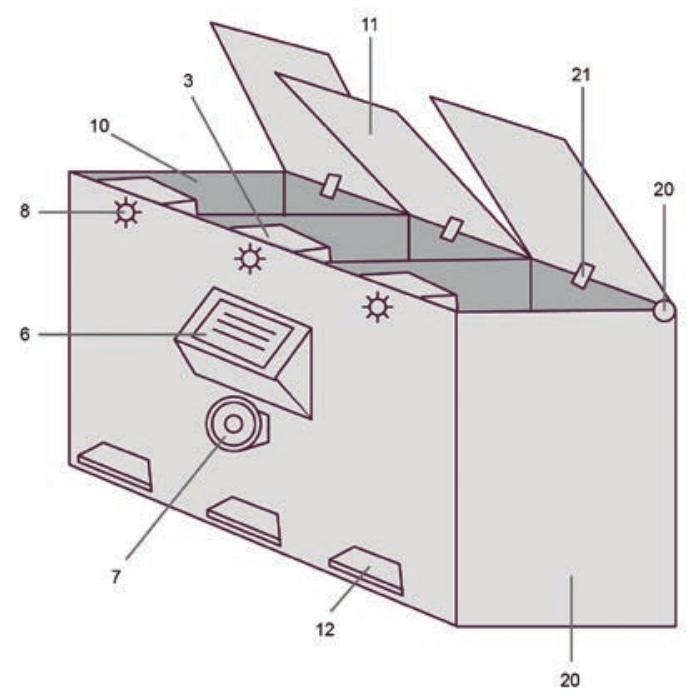

Fuente: Elaboración propia. 
Figura 2. Maqueta de la PRA de Classyfied

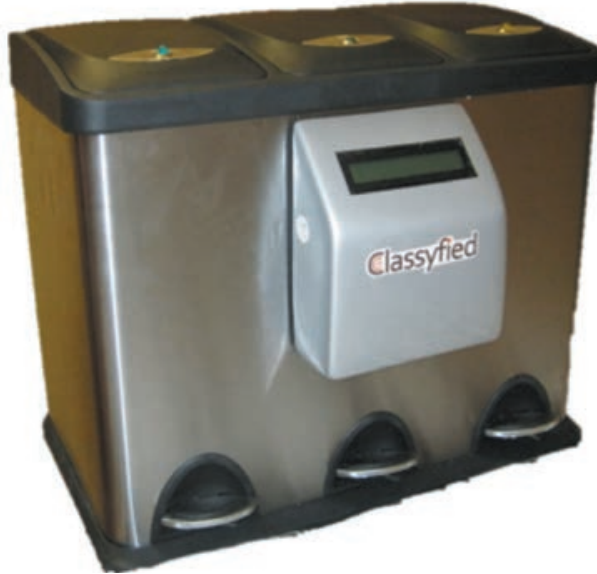

Fuente: Elaboración propia.

En las figuras 1 y 2 se puede observar un esbozo del diseño, así como el primer prototipo de la papelera de reciclaje con reconocimiento automático de productos (PRA). Su funcionamiento es muy sencillo: cuando un usuario se dispone a reciclar un envase, pulsa el pedal (12) del compartimento de reciclaje que corresponde. En caso de dudas, puede acercar el envase a la pantalla (6), que lo detectará y mostrará por pantalla el tipo de reciclaje que se debe realizar, encendiendo la luz LED del compartimento correspondiente (8). El producto, al entrar en el compartimento, será detectado por el lector de RFID (3). Si el producto es reciclado de forma incorrecta, se informará al usuario mediante una notificación sonora (7) configurable, así como con indicaciones visuales que se mostrarán por pantalla (6). Una vez reciclado, los datos recopilados serán enviados a los servidores de Classyfied utilizando la red Wi-Fi doméstica del usuario o, en el caso de no existir, mediante una conexión 3G.

Servicios de Classyfied: Valor añadido a toda la cadena de distribución

La información obtenida por la PRA representa la principal ventaja competitiva de la idea de negocio, dado que, hoy en día, ningún competidor puede obtener estos datos con el mismo nivel de calidad que Classyfied. Mediante estos la empresa construirá sus servicios, expuestos a continuación desglosados en función del público destinatario (figura 3).

Figura 3. Cadena de valor de Classyfied

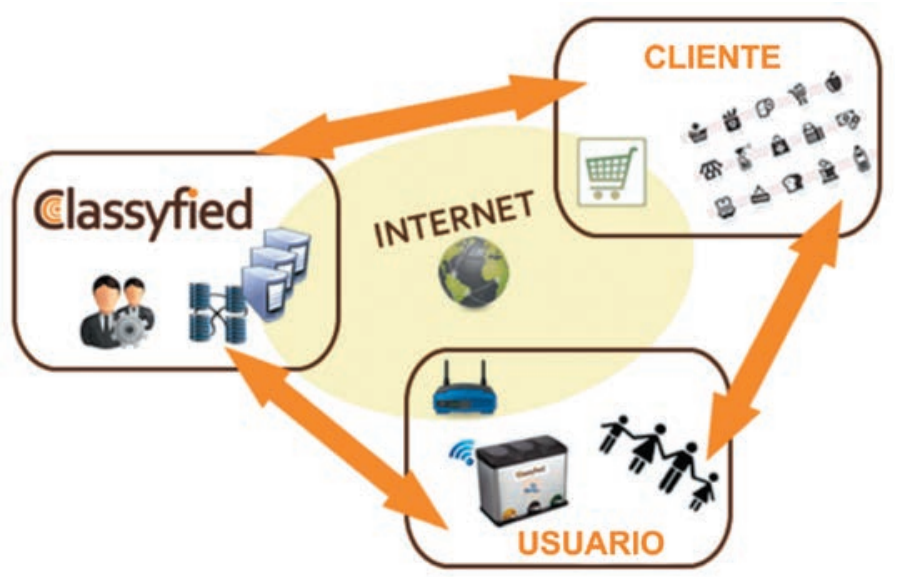

Fuente: Elaboración propia. 
Gracias a Classyfied, los usuarios finales, es decir, compradores y consumidores finales de los productos vendidos por los supermercados, podrán:

- Contar con un proceso automático de reposición de productos por el simple hecho de utilizar la PRA. El supermercado del usuario recibirá instantáneamente los datos de los productos consumidos al ser detectados por la PRA y generará de forma autónoma una lista de la compra con estos. El usuario solo tendrá que configurar la frecuencia de reposición y elegir el momento del día en el que quiere recibir la compra de nuevo en su domicilio, olvidándose de tener que volver a hacer la compra y consiguiendo así liberar hasta tres horas semanales de tiempo libre.

- Beneficiarse también de un marketing más personalizado por parte de su supermercado, recibiendo ofertas y promociones relacionadas con los productos consumidos en función del patrón de consumo que se desarrollará sobre la base de los hábitos observados.

- Contar con un asistente en el reciclado, que los ayudará a realizar la separación de basuras de forma correcta gracias a las indicaciones visuales y sonoras que incorpora la PRA.

Las ventajas que aporta Classyfied a sus clientes, supermercados con venta en el canal en línea, son diversas:

- En primer lugar, los supermercados dispondrán de datos sobre el consumo de los productos previamente vendidos. Con estos datos y la ayuda de Classyfied podrán optimizar los procesos de venta y distribución de productos, ofreciendo a sus clientes (consumidores) el servicio de reposición automática de productos comentado previamente, lo que les permite asegurar una cifra de ventas anual (cliente cautivo) y un incremento de la tasa de retención y la fidelidad de sus clientes, así como de su satisfacción.

- La información obtenida por la papelera de reciclaje permite a Classyfied proveer a los supermercados de un servicio de marketing totalmente personalizado para cada usuario final con un nivel de detalle nunca antes alcanzado, que permite ofrecer el producto adecuado a la persona apropiada en el momento preciso. Este servicio redundará en mayores beneficios gracias a su alta eficacia (superior tasa de éxito y mayores ventas por mejor adaptación al perfil del usuario) y bajo coste.

- Finalmente, un servicio adicional ofrecido por Classyfied a los supermercados es la optimización de sus inventarios. El conocimiento sobre los patrones de consumo de los usuarios finales permitirá incorporar un nuevo modelo de gestión innovador y totalmente optimizado de los almacenes, ya que pueden conocer la cantidad exacta de estos necesitada en cada momento de tiempo con un grado de precisión nunca antes alcanzado, lo que reduce los costes de inventario a menos de la mitad respecto a la actualidad.

Gracias a las características de Classyfied se podrán ofrecer igualmente servicios a terceros:

- Así, las instituciones públicas pueden convertirse en un cliente de Classyfied patrocinando la empresa a cambio de información real sobre el volumen de basuras generado, porcentajes de productos mal reciclados, etc. Con esta información, los ayuntamientos, consejerías de Medio Ambiente o empresas del sector podrán enfocar sus esfuerzos de fomento del reciclaje en las necesidades reales de los ciudadanos.

- Cuando esté implementado en nuestro país el sistema de depósito, devolución y retorno (SDDR) (7), Classyfied podrá encargarse de contabilizar los envases devueltos y descontar directamente de la compra el depósito fijado.

- Dado el alto nivel de detalle de la información recopilada por la PRA, Classyfied podrá elaborar bajo demanda informes de mercado para productores de alimentos y bebidas, empresas de consultoría, etc. Esto permitirá comparar, por ejemplo, el consumo por barrios o calles entre un producto y su competidor. 


\section{Ingresos de Classyfied}

Aunque una idea de negocio sea excepcional, si no se monetiza y rentabiliza no será viable a largo plazo. Por ello, Classyfied presenta un sólido modelo económico con diferentes fuentes de ingresos. La más importante en los primeros años será la venta de PRA. Las papeleras estarán diseñadas y fabricadas por la propia empresa y tendrán un precio aproximado de sesenta euros. Se trata de un ingreso no recurrente, con un margen sobre los costes totales de aproximadamente un $20 \%$ en el inicio.

Sin embargo, los ingresos que están llamados a liderar la cuenta de resultados de Classyfied son aquellos provenientes de los servicios ofertados a los supermercados asociados. La empresa facturará una comisión del $1,7 \%$ por las ventas realizadas a través de la PRA. Esta es una fuente de ingresos recurrente, con un margen que se elevará a medida que la empresa incremente su tamaño y eficiencia. Adicionalmente, la empresa contará con otras fuentes de ingresos de menor peso e importancia, como son la consultoría de optimización de almacenes, la venta de informes de mercado, etc.

\section{Implementación}

Los riesgos asociados al lanzamiento al mercado de un producto innovador como es la PRA de Classyfied son muy elevados. Con el fin de reducirlos, resultará fundamental en fases previas al establecimiento de la compañía el disponer de un preacuerdo con un cliente de lanzamiento. Este supermercado tendrá la exclusividad de los servicios de Classyfied durante un determinado tiempo y será el único en ofrecer el sistema de reposición automática de productos a sus clientes, ventaja competitiva principal que ofrece la empresa. Adicionalmente, se parte de la hipótesis de mantener aprobado un préstamo participativo con la Empresa Nacional de Innovación, S. A. (ENISA).

Una vez alcanzados estos dos retos, se podrán iniciar los trámites legales para la puesta en marcha de la empresa, incluyendo el alta en los registros pertinentes, registro de marca y nombre comercial, reclamar la patente en mercados que pueden resultar interesantes en el futuro (Europa y Estados Unidos), etc. En paralelo, se buscarán y alquilarán las instalaciones industriales de la empresa y se establecerán las oficinas de la empresa.

En una posterior fase se iniciará la producción de las primeras PRA y se desarrollará el sistema informático que interconectará Classyfied con el cliente de lanzamiento. En este punto será fundamental realizar una prueba piloto con usuarios finales reales con el fin de corregir posibles errores e introducir mejoras en los procesos. Una vez superada esta prueba, se iniciará la comercialización de las PRA de Classyfied a los usuarios finales y comenzarán las operaciones de la compañía.

Para soportar este despliegue, se ha desarrollado un plan financiero que plantea llegar hasta los 16.000 usuarios finales al final del tercer año de operaciones, considerando un consumo medio anual por familia en productos de alimentación ligeramente superior a los 2.000 euros. Se trata de una cifra conservadora pero realista considerando que solo en el 2014, en las ciudades de más de 100.000 habitantes el porcentaje de familias que realizaron compra en línea de productos de alimentación se situó cerca del 10\% y el gasto medio per cápita en este tipo de producto fue de 1.500 euros (1). Bajo estos supuestos, Classyfied ofrece unos resultados económicos y financieros muy positivos: a partir del segundo año se supera el punto muerto y se obtienen beneficios netos, que permitirán financiar la posterior expansión de la empresa.

\section{Conclusiones}

Classyfied ofrece propuestas claras, mediante un producto disruptivo que puede revolucionar el modo como los consumidores se relacionan con los supermercados y adquieren los productos de alimentación. Se trata, 
además, de una innovación alineada con sectores que están en plena expansión, como es el comercio en línea, el internet de las cosas o la mayor conciencia por el reciclaje.

El TFG desarrollado profundiza en la viabilidad tanto técnica como económica del modelo de negocio que explotar y deja la puerta abierta a un desarrollo real de este. Los primeros pasos para materializar la idea irán encaminados a realizar contactos comerciales con potenciales clientes con el fin de realizar demostraciones de concepto y encontrar un socio de lanzamiento. En paralelo, será fundamental continuar con la investigación y el desarrollo de nuevos modelos de PRA más avanzados. Si todo lo anterior evoluciona favorablemente, será el momento de concretar la financiación con el fin de hacer realidad el proyecto.

\section{Bibliografía}

1. MINISTERIO DE MEDIO AMBIENTE RURAL Y MARINO (2014). «Estudio de mercado - Observatorio del Consumo y la Distribución Alimentaria» [artículo en línea]. [Fecha de consulta: 8 de noviembre de 2014].

2. INSTITUTO NACIONAL DE ESTADÍSTICA (2011). «Encuesta de Empleo del Tiempo 2009-2010» [artículo en línea]. [Fecha de consulta: 2 de octubre de 2014].

3. INSTITUTO NACIONAL DE ESTADÍSTICA (2013). «Encuesta de Presupuestos Familiares 2013» [artículo en línea]. [Fecha de consulta: 18 de octubre de 2014].

4. KANTAR WORLD PANEL (2014). «Balance 2013 del sector del Gran Consumo» [artículo en línea]. [Fecha de consulta: 12 de octubre de 2014].

5. MCKINSEY GLOBAL INSTITUTE (2015). «Unlocking the potential of the Internet of Thing» [artículo en línea]. [Fecha de consulta: 30 de junio de 2015].

6. INSTITUTO NACIONAL DE ESTADÍSTICA (2012). «Estadísticas sobre la recogida y tratamiento de residuos - Encuesta sobre generación de residuos urbanos» [artículo en línea]. [Fecha de consulta: 20 de octubre de 2014].

7. Ley 22/2011, de 28 de julio, de Residuos y Suelos Contaminados. Boletín Oficial del Estado (29 de julio de 2011), pág. 85650-85705.

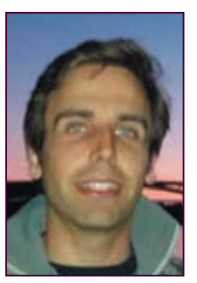

\section{Daniel Macías Perea}

\section{Alumni Grado ADE (UOC)}

Ingeniero en Aviación Civil por la Ecole Nationale de l'Aviation Civile francesa e informático por la Facultad de Informática de Barcelona de la Universidad Politécnica de Cataluña. MBA Aeroespacial por la Escuela de Organización Industrial y graduado en Administración y Dirección de Empresas por la UOC. Su carrera profesional se ha desarrollado como gestor de proyectos tecnológicos en el ámbito de la industria aeronáutica en empresas como Indra y Airbus y, más recientemente, en el campo de los recursos humanos en la aerolínea Iberia.

Los textos publicados en esta revista están -si no se indica lo contrario- bajo una licencia Reconocimiento-Sin obras derivadas 3.0 España de Creative Commons. Puede copiarlos, distribuirlos y comunicarlos públicamente siempre que cite su autor y la revista y la institución que los publica (autoría, nombre de la revista, institución editora); no haga con ellos obras derivadas. La licencia completa se puede consultar en http://creativecommons.org/licenses/by-nd/3.0/es/deed.es.

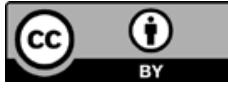

